UCTP101.02

\title{
Non-Vanishing Cosmological Constant $\Lambda$, Phase Transitions, And $\Lambda$-Dependence Of High Energy Processes
}

\author{
Freydoon Mansourit \\ Physics Department, University of Cincinnati, Cincinnati, OH 45221
}

\begin{abstract}
It is pointed out that a collider experiment involves a local contribution to the energy-momentum tensor, a circumstance which is not a common feature of the current state of the Universe at large characterized by the cosmological constant $\Lambda_{0}$. This contribution may be viewed as a change in the sturcture of space-time from its large scale form governed by $\Lambda_{0}$ to one governed by a $\Lambda$ peculiar to the energy scale of the experiment. Possible consequences of this effect are explored by exploiting the asymptotic symmetry of space-time for non-vanishing $\Lambda$ and its relation to vacuum energy.
\end{abstract}

*e-mail address: mansouri@uc.edu 


\section{1 introduction}

The notion of phase transition plays an important role in our understanding of particle physics at the fundamental level. It also plays an important role in the inflationary cosmology. One may therefore view this notion as an important factor in shaping our ideas of the Universe today. Its most concrete realization has been through spontaneously broken symmetry. For whatever dynamical reasons, when a phase transition takes place, there will be a contribution to the vacuum energy density $\rho_{\text {vac }}$ of space-time. On the basis of Einstein's field equations, such a non-vanishing vacuum energy is equivalent to having a cosmological constant, $\Lambda$, given by [1], 2]

$$
\Lambda=8 \pi G_{N} \rho_{v a c}
$$

where $G_{N}$ is Newton's constant. This relation raises a number of interesting issues. Among these, the one that has received a good deal of attention in recent years is that, the contribution to $\Lambda$ from each phase transition, such as that at the electroweak scale, is very large [1, 2] according to the current theories. It follows that unless a delicate cancellation mechanism such as supersymmetry has been in operation over the entire range of scales from the time of big bang to present, it would be difficult to account for the smallness of $\Lambda_{\text {observed }}$ according to standard interpretations. On the other hand, if we view the vacuum energy arising from phase transitions as the primary source of $\Lambda$, a constant $\Lambda$ is not compatible with such phase transitions.

Another issue which arises from the equality given by Eq. (1) is that for finite $\Lambda$, the asymptotic symmetry group of space-time is not Poincaré but de Sitter $(\mathrm{d} S)$ or anti-de Sitter (AdS) group. On the other hand, the well known Einstein massenergy relation $E=m c^{2}$ is a consequence of Poincaré symmetry and is valid only in Minkowski space-time. One of the aims of the present work is to point out how the rest energy expression is modified in $\mathrm{dS}_{4}$ and $\mathrm{AdS}_{4}$ spaces and how they depend on the cosmological constant and the vacuum energy. As mentioned above, if we take the connection between $\Lambda$ and phase transitions seriously, there must have been some periods in the history of the Universe, at which the value of $\Lambda$ changed significantly because of phase transitions. In particular, at some such periods, the value of $\Lambda$ must have been very large. It would be of interest to see how the kinematics of high energy reactions were modified during such periods and whether this led to any testable consequences. It will be seen below that if again we take the phase transition, or its realization as spontaneously broken symmetry, as the primary source of a non-vanishing $\Lambda$, it may be possible to test some of the consequences at the next generation of high energy colliders. To accomplish this, it will be necessary to reexamine the relation between the processes in the current high energy experiments and the period (energy scale) in the history of the Universe at which such processes occurred routinely. The consequences will be significant [3] if a high energy experiment modifies the local structure of space-time for a short period of time, so that the immediate neighborhood of a collision departs from the Minkowski space and becomes 
a dS or AdS space-time. In both $\mathrm{dS}$ and AdS spaces the ground state energies, i.e., the analogs of the energy in Einstein's mass-energy relation, depend not only on the mass but also on spin and on $\Lambda$, with the correct value of $\Lambda$ to be determined such that it is appropriate to the relevant energy scale.

The mathematical frameworks for obtaining the analogs of Einstein's mass-energy relation in $\mathrm{AdS}_{4}$ and $\mathrm{dS}_{4}$ spaces depend on a mixture of the representation theory of the corresponding groups and the field theoretic constructions in these spaces. Most of this already exist in the literature in one form or another. The construction of the representations for these groups relies on the structure of appropriate little groups. For $\mathrm{AdS}_{4}$ group, the relevant little group is [4, 5, 6] the compact group $S O(2) \times S O(3)$, and it allows for a simple identification of an appropriate energy operator. For the $\mathrm{dS}_{4}$ group, the simplest choice for a subgroup is the maximal compact subgroup [7] $S O(4)$. However, when the representations are induced in this way, the direct identification of one of the generators with an energy operator does not lead to a physically suitable energy spectrum. So, we will use an alternative realization of the algebra due to Bohm [8]. In both spaces, to relate the eigenvalues of the quadratic Casimir operators to the physical masses, it is necessary to introduce the concept of mass as the pole of the propagator in an appropriate quantum field theory. To lowest order this pole would be that of the Green's function for a $\mathrm{dS}_{4}$ ( or $\mathrm{AdS}_{4}$ ) invariant differential equation. As is familiar from field theories in Minkowski space time, it is desirable that such an equation become conformally invariant in the limit of vanishing mass [9, 10]. Combining these concepts, and assuming the existence of an invariant vacuum state [9, 11, 12], it is then possible to obtain an expression for the ground state energy. In carrying out this project, one must also carefully keep track of the dependence on the cosmological constant, $\Lambda$. In the discussions of the representation theories of $\mathrm{AdS}$ and $\mathrm{dS}$ groups mentioned above, the explicit values of the radii of curvatures of the corresponding spaces play no essential roles, so that they are often set equal to unity. In view of Eq. (1) and the point of view emphasized in this work, to extract physical consequences it is essential that we explicitly display the dependence on this dimensional parameter or, equivalently, the cosmological constant.

The change in the asymptotic structure of space-time from Minkowski to dS or AdS spaces implies a change in the asymptotic symmetry group of space-time. In particular, it implies the breakdown of translational invariance. In the last decade, possible violations of Lorentz and CPT symmetries have been studied in great detail [13]. In the same vein, the results presented in this work may also be viewed as probing the possible violation of translational invariance.

\section{The dS and AdS Spaces and Algebras}

We begin with some of the well known properties of these spaces and algebras. The $\mathrm{dS}$ and AdS spaces and algebras have many features in common. So, we give the details for one of them and then indicate the changes, if any, in the corresponding 
expressions for the other space and algebra. As much as possible, we will follow the notation and conventions of reference [14].

The anti-de Sitter space in $3+1$ dimensions can be viewed as a subspace of a flat 4-dimensional space with the line element

$$
d s^{2}=d X_{A} d X^{A}=d X_{0}^{2}-d X_{1}^{2}-d X_{2}^{2}-d X_{3}^{2}+d X_{4}^{2}
$$

It is determined by the constraint

$$
\left(X_{0}\right)^{2}-\left(X_{1}\right)^{2}-\left(X_{2}\right)^{2}-\left(X_{3}\right)^{2}+\left(X_{4}\right)^{2}=l^{2}
$$

where $l$ is a real constant and is related to the cosmological constant according to $\Lambda=-l^{-2}$. The set of transformations which leave the line element invariant form the $\mathrm{AdS}_{4}$ group $S O(3,2)$. We will actually deal with the universal cover of this group.

Similarly, the $\mathrm{dS}_{4}$ space can be viewed as a subspace of a flat 4-dimensional space with line element

$$
d s^{2}=d X_{A} d X^{A}=d X_{0}^{2}-d X_{1}^{2}-d X_{2}^{2}-d X_{3}^{2}-d X_{4}^{2}
$$

It is determined by the constraint

$$
\left(X_{0}\right)^{2}-\left(X_{1}\right)^{2}-\left(X_{2}\right)^{2}-\left(X_{3}\right)^{2}-\left(X_{4}\right)^{2}=-l^{2},
$$

where now $\Lambda=l^{-2}$.

The $\mathrm{AdS}_{4}$ algebra consists of the elements $M_{A B}$ satisfying the commutation relations

$$
\left[M_{A B}, M_{C D}\right]=i\left(\eta_{A D} M_{B C}+\eta_{B C} M_{A D}-\eta_{A C} M_{B D}-\eta_{B D} M_{A C}\right) .
$$

With the index $A=(\mu, 4)$ and $\mu=0,1,2,3$, we can write the algebra in a more familiar four dimensional notation by setting

$$
M_{\mu 4}=l \Pi_{\mu}
$$

With the notation

$$
\epsilon^{0123}=1 ; \quad \eta^{a b}=(1,-1,-1,-1)
$$

we get

$$
\begin{gathered}
{\left[M_{\mu \nu}, M_{\rho \sigma}\right]=i\left(\eta_{\mu \sigma} M_{\nu \rho}+\eta_{\nu \rho} M_{\mu \sigma}-\eta_{\mu \rho} M_{\nu \sigma}-\eta_{\nu \sigma} M_{\mu \rho}\right)} \\
{\left[M_{\mu \nu}, \Pi_{\rho}\right]=i\left(\eta_{\nu \rho} \Pi_{\mu}-\eta_{\mu \rho} \Pi_{\nu}\right)} \\
{\left[\Pi_{\mu}, \Pi_{\nu}\right]=-i l^{-2} M_{\mu \nu}=i \Lambda M_{\mu \nu} .}
\end{gathered}
$$

As expressed in terms of the cosmological parameter $\Lambda$, this algebra also holds for $\mathrm{dS}_{4}$. It can be seen that, under suitable conditions, in the limit of vanishing $\Lambda$, the $\mathrm{AdS}_{4}$ and $\mathrm{dS}_{4}$ algebras contract to the Poincaré algebra. 
The $\mathrm{AdS}_{4}$ and $\mathrm{dS}_{4}$ groups have each two Casimir invariants. The quadratic ones are given, with appropriate signs for $\Lambda$, by

$$
I_{1}=-\frac{\Lambda}{2} M_{A B} M^{A B}=\Pi_{\mu} \Pi^{\mu}-\frac{\Lambda}{2} M_{\mu \nu} M^{\mu \nu}
$$

The important point here is that, in contrast to Poincaré algebra, for $\mathrm{AdS}_{4}$ as well as $\mathrm{dS}_{4}$ algebras the quantity $\Pi_{\mu} \Pi^{\mu}$ is not an invariant by itself. As a result, the analogs of Einstein mass-energy relation $E=m c^{2}$ will also depend on spin and the cosmological constant, as we shall see below.

The second Casimir invariant $I_{2}$ for either $\mathrm{AdS}_{4}$ or $\mathrm{dS}_{4}$ algebras can be written as

$$
I_{2}=\frac{\Lambda}{16} V_{A} V^{A}
$$

where

$$
V^{A}=\epsilon^{A B C D E} M_{B C} M_{D E} .
$$

Defining the analog of the Pauli-Lubanski operator as

$$
W^{\mu}=V^{\mu}=\epsilon^{\mu \nu \rho \sigma} \Pi_{\nu} M_{\rho \sigma},
$$

we can write $I_{2}$ in four dimensional notation for both algebras:

$$
I_{2}=W_{\mu} W^{\mu}-\frac{\Lambda}{16}\left(V^{4}\right)^{2}
$$

\section{Massive Unitary Representations}

It will be recalled that for the Poincaré group, the construction of unitary representations begins by identifying an appropriate little group. For massive momenta, e.g., the little group is $S O(3)$. Then the corresponding induced representation would be specified by the ground state eigenvalues of the Casimir operators, i.e., by the (rest) energy $E_{0}$ and spin $s$. The quantity $E_{0}^{2}$ is thus the ground state eigenvalue of the quadratic Casimir operator $P_{\mu} P^{\mu}$ of the Poincaré algebra. The notion of mass is then introduced as the pole of the propagator in the corresponding field theory. For the Poincaré group, this equation is the relativistically invariant free massive Klein-Gordon equation. Then, noting that the d'Alembertian operator is just the representation of the Casimir operator $P_{\mu} P^{\mu}$ in terms of differential operators, one obtains:

$$
\left[P_{\mu} P^{\mu}-m^{2} c^{4}\right] \phi=0
$$

This provides a group theoretic basis for obtaining the expression $E_{0}=m c^{2}$. One can follow the same recipe for relating the notions of mass and energy in $\mathrm{dS}_{4}$ and $\mathrm{AdS}_{4}$ spaces. To this end, we must first obtain the ground state eigenvalues of the corresponding quadratic Casimir operators. We begin with $\mathrm{AdS}_{4}$. 
The massive discrete unitary representations for $\mathrm{AdS}_{4}$ algebra has been known for sometime [5, 6]. With $S O(2) \times S O(3)$ as maximal compact subgroup, let

$$
H=l \Pi_{0} ; \quad J_{ \pm}=M_{23} \pm i M_{31} ; \quad J_{3}=M_{12}
$$

The J's form the algebra of the $S O(3)$. They commute with $H$ which generates the $S O(2)$ part. The quantity $\Pi^{\mu}$ has the same dimension as $P^{\mu}$ in the Poincaré algebra, so that it is natural to identify $\Pi^{0}$ as the energy operator. The generator $H$ is then the dimensionless version of the energy operator. So, we can label our states by the eigenvalues of one or the other of these operators.

We can choose the remaining six generators of $\mathrm{AdS}_{4}$ algebra such that they can act as raising and lowering operators for eigenvalues of $\Pi^{0}$. To this end, let

$$
B_{i}^{ \pm}=M_{0 i} \pm i l \Pi_{i} ; \quad i=1,2,3 .
$$

All three of the plus (minus) operators raise (lower) the eigenvalues of $H$ :

$$
\left[H, B_{i}^{ \pm}\right]= \pm B_{i}^{ \pm}
$$

In this $\{H, J, B\}$ basis, the quadratic Casimir operator will take the form

$$
I_{1}=\Pi_{0}\left(\Pi_{0}-\frac{3}{l}\right)+\frac{1}{l^{2}} \vec{J}^{2}+\frac{1}{l^{2}} B_{i}^{+} B_{i}^{-}
$$

where in the last term a sum over the index " $\mathrm{i}$ " is to be taken.

Using the above preparations, we can now label [5, 6] the discrete representations of $\mathrm{AdS}_{4}$ algebra by the labels induced by subgroup $S O(2) \times S O(3)$. Depending on whether we use eigenvalues of $\Pi_{0}$ or $H$, they are:

$$
\left|E, j, j_{3}, E_{\Lambda}>; \quad\right| h, j, j_{3}, E_{\Lambda}>
$$

The quantity $E_{\Lambda}$ is the energy scale associated with the value of $\Lambda$ or, equivalently, with the radius of curvature $l$. For these unitary representations, we denote the lowest (ground state) eigenvalues by $E_{0}$ and $s$, respectively. This means, in particular, that

$$
B_{i}^{-} \mid E_{0}, s, s_{3}, E_{\Lambda}>=0 .
$$

Applying the quadratic Casimir operator on a ground state, we get

$$
I_{A d S}\left|E_{0}, s, s_{3}, E_{\Lambda}>=\left[E_{0}\left(E_{0}-3 E_{\Lambda}\right)+E_{\Lambda}^{2} s(s+1)\right]\right| E_{0}, s, s_{3}, E_{\Lambda}>,
$$

Comparing this expression with the corresponding eigenvalue for the Poincaré algebra, it is already clear that the ground state energy $E_{0}$ will depend on $s$ and on $E_{\Lambda}$.

Next, consider the $\mathrm{dS}_{4}$ algebra. In this case, the maximal compact subgroup is $S O(4)$. Its labels can be used to induce and classify the unitary representations of the $\mathrm{dS}_{4}$ algebra [7]. To construct an appropriate energy-momentum operator, however, 
we will make use of a representation of $\mathrm{dS}_{4}$ algebra pioneered by Bohm [8], in which the unitary principal series of $\mathrm{dS}_{4}$ are realized on an identical pair of positive energy representations of the Poincaré group. Both the Poincaré and $\mathrm{dS}_{4}$ groups contain the Lorentz group as a subgroup. The corresponding generators, $M_{\mu \nu}$ satisfy the commutation relations given in Eq. (9). Let $P_{\mu}$ represent the (commuting) translation generators of the Poincaré algebra, so that its quadratic Casimir operator is given by $P^{2}=P_{\mu} P^{\mu}$. Then, using a notation similar to that for $\mathrm{AdS}_{4}$ given above, let

$$
\Pi_{\mu}=P_{\mu}+\left[\frac{\Lambda}{2 P^{2}}\right]^{1 / 2}\left\{P^{\nu}, M_{\nu \mu}\right\}
$$

where, as in previous section, $\Lambda=l^{-2}$ is the cosmological constant. It is easy to check that the $\Pi_{\mu}$ 's defined in this way satisfy the commutation relations given in Eq. (9). Therefore, we have a representation of the $\mathrm{dS}_{4}$ algebra in terms of the operators $P_{\mu}$ and $M_{\mu \nu}$.

In this representation of $\mathrm{dS}_{4}$ algebra, the quadratic Casimir operator of the algebra given by Eq. (10) can be expressed as

$$
I_{1}=P_{\mu} P^{\mu}+\frac{9}{4} E_{\Lambda}^{2}+E_{\Lambda}^{2} \omega_{\mu} \omega^{\mu} .
$$

In this equation, the quantity

$$
\omega_{\mu}=\frac{1}{2} \epsilon_{\mu \nu \rho \sigma} P^{\nu} M^{\rho \sigma}
$$

is the Pauli-Lubanski operator of the Poincaré algebra. Also, as in the case of $\mathrm{AdS}_{4}$ algebra, we have replaced the cosmological constant with the corresponding energy scale $E_{\Lambda}$.

We can now compute the eigenvalues of $I_{1}$ acting on positive energy representations of the Poincaré group. We label the Poincaré state with its ground state (rest) energy $E_{0}$ and its spins $s$. Thus we have

$$
I_{d S}\left|E_{0}, s, E_{\Lambda}>=\left[E_{0}^{2}+E_{\Lambda}^{2}\left(\frac{9}{4}-s^{2}-s\right)\right]\right| E_{0}, s, E_{\Lambda}>.
$$

The range of values of $s$ is the same as one of labels for the unitary principal series representations of the $\mathrm{dS}_{4}$ group, so that we can take $s$ as a suitable definition of spin in $\mathrm{dS}_{4}$ space. Similarly, the operator $P^{0}$ has the correct dimension and the range of eigenvalues to be identified as the energy operator. We note, however, that, in contrast to Poincaré algebra, since $P_{\mu} P^{\mu}$ is not an invariant of $\mathrm{dS}_{4}$ algebra, $E_{0} \neq m c^{2}$ in $\mathrm{dS}_{4}$ space. The connection to mass will be discussed in the next section.

\section{$4 \Lambda$-Dependence of Mass-Spin-Energy Relations}

We now turn to the issue of how the notion of mass arises in $\mathrm{dS}_{4}$ or $\mathrm{AdS}_{4}$ spaces and how it gets related to the eigenvalues of their respective quadratic Casimir operators. 
In a standard field theory, the mass of a particle is identified as the pole of the propagator for the corresponding field. To lowest order, the propagator is the classical Green's function for an invariant differential equation. For a scalar field in Minkowski space, e.g., it is the Green's function for the Klein-Gordon equation

$$
\left(\partial^{\mu} \partial_{\mu}+m^{2}\right) \phi(x)=0
$$

This equation immediately generalizes to curved space-time by replacing $\partial_{\mu}$ with an appropriate covariant derivative $\nabla_{\mu}$ :

$$
\left(\nabla_{\mu} \nabla^{\mu}+m^{2}\right) \phi(x)=0 .
$$

It has been observed, however, that in contrast to Klein-Gordon equation the massless limit of this equation is not conformally invariant [9, 10. If we require, in analogy with the corresponding situation in Minkowski space, that the massless limit of the relevant differential equation be conformally invariant, we must modify this equation by a term proportional to the scalar curvature [9, 10]:

$$
\left(\nabla_{\mu} \nabla^{\mu}+\frac{R}{3}+m^{2}\right) \phi(x)=0 .
$$

where for $\mathrm{dS}_{4}$ and $\mathrm{AdS}_{4}$ spaces,

$$
R= \pm \frac{3}{2 l^{2}}
$$

More generally, for a particle of any spin, we have

$$
\left(\nabla_{\mu} \nabla^{\mu}-E_{\Lambda}^{2} \beta_{s}+m^{2} c^{4}\right) \Psi_{s}=0
$$

where $\beta_{s}$ is a rational number that can be different for different spin. It is to be noted that the question of whether or not the massless limit of a wave equation in curved space should have the same symmetry, i.e., conformal invariance, as that of the corresponding equation in Minkowski space, must ultimately be settled by its physical predictions. As far as the result given below are concerned, conformal invariance or lack thereof affects the value of the coeffeciant $\beta_{s}$.

With a suitable differential equation at hand, it is now straight forward to connect it to the quadratic Casimir operator, $I_{1}$, of the $\mathrm{dS}_{4}$ or $\mathrm{AdS}_{4}$ algebra. This can be worked out in a manner which is familiar from the relation indicated in the previous section between the d'Alembertian operator and the quadratic Casimir operator of the Poincaré (Lie) algebra in Minkowski space. This means that to have an invariant differential equation, we must have

$$
I_{1}=-\nabla_{\mu} \nabla^{\mu}+b(s) .
$$

where the quantity $b(s)$ can depend on spin. Using the field equation and the eigenvalues for $I_{1}$ of $\mathrm{dS}_{4}$ given by Eq. (26), we get

$$
m^{2} c^{4}=E_{0}^{2}+E_{\Lambda}^{2}\left(\frac{9}{4}-s^{2}-s+\beta_{s}\right)-b(s) .
$$


Similarly, using Eq. (22) for $\mathrm{AdS}_{4}$, we get, with in general a different value for $\beta_{s}$,

$$
m^{2} c^{4}=E_{0}\left(E_{0}-3 E_{\Lambda}\right)+E_{\Lambda}^{2}\left(s^{2}+s+\beta_{s}\right)-b(s) .
$$

The precise form of $b(s)$ will depend on the choice of unitary representations and other constraints. For example, for the discrete unitary series of $\mathrm{AdS}_{4}$, one can use the non-negativity of the norms of the states [5] to get

$$
m^{2} c^{4}=E_{0}\left(E_{0}-3 E_{\Lambda}\right)+E_{\Lambda}^{2}\left(2+s-s^{2}\right) .
$$

What is important for our purposes is that both for $\mathrm{dS}_{4}$ and $\mathrm{AdS}_{4}$ the expression for the ground state energy is of the form

$$
E_{0}=E_{0}\left(m, s, E_{\Lambda}\right)
$$

\section{Potential Experimental Consequences and Spec- ulations}

One of the straight forward but important consequences of a non-vanishing cosmological constant is that it changes the asymptotic symmetry group of space-time from Poincaré group to de Sitter or anti-de Sitter group. One immediate consequence of this is that the familiar Einstein rest energy expression $E_{0}=m c^{2}$ is modified to an expression given by Eq. (36), involving mass, spin, and an energy scale determined by the cosmological constant. Moreover, if our current theories of particle physics based on spontaneously broken symmetry and phase transition are correct, then there must have been some periods in the history of the Universe, in which the value of $\Lambda$, and hence of $E_{\Lambda}$, changed significantly. This is clearly not compatible with the idea that a single constant $\Lambda$, vanishing or non-vanishing, has controlled the expansion of the Universe. It thus appears that a suitable theoretical framework would have to regard $\Lambda$ as a dynamical quantity, which is both space and time dependent. The explicit construction of such a framework is beyond the scope of this work. Here, we confine the discussion to some general remarks on the $\Lambda$-dependence of high energy processes.

Let us consider one of the potential experimental consequences of the expression given by Eq. (36). To this end, it will be recalled that the quantity $E_{\Lambda}$ is the energy associated with the radius of curvature, $l_{\Lambda}$, of the $\mathrm{AdS}_{4}$ or $\mathrm{dS}_{4}$ space. This energy scale is related to but is distinct from the scale at which the vacuum energy is evaluated. The difference between the two has to do with the appearance of Newton's constant $G$ in Eq. (1). As a first try, let us assume, somewhat naively, that it is the current value $\Lambda_{0}$ of the cosmological parameter that should set the energy scale $E_{\Lambda}$. Then, given the current bounds [1, 2],

$$
l_{\Lambda_{0}}=\left|\Lambda_{0}\right|^{-\frac{1}{2}} \sim 10^{28} \mathrm{~cm} .
$$


It follows that

$$
E_{\Lambda_{0}} \sim 10^{-33} \mathrm{eV}
$$

So, if the relevant energy scale $E_{\Lambda}$ in Eq. (36) were set by the current value of the cosmological parameter, the deviation from $E=m c^{2}$ would be very small, and there would be no hope for the experimental detection of such deviations in the existing or the planned future colliders.

On the other hand, if our current theories of particle physics based on spontaneously broken symmetry and phase transition are correct, then there must have been some periods in the history of the Universe, in which the value of $\Lambda$, and hence of $E_{\Lambda}$, were large. For example, in the electroweak epoch characterized by $\Lambda_{E W}$, the kinematics of a typical electroweak process must have depended significantly on $E_{\Lambda_{E W}}$. It is then of interest to see whether this kind of $\Lambda$-dependence can be detected in high energy collicer experiments. In this respect, we note that a typical high energy collider experiment at, say, $20 \mathrm{TeV}$ does not necessarily involve a phase transition leading to a significantly larger value of $\Lambda$. But it is conceivable that in future collider machines a mechanism could be devised such that, in the immediate neighborhood of the collision, one can justify the connection between the scale of the experiment and the value of $\Lambda$ corresponding to a phase transition at that scale. Assuming such a connection, let us compute the expected order of magnitude deviations from $E=m c^{2}$ law.

For energies of the order of $200 \mathrm{GeV}$ corresponding to the electroweak phase transition, the radius of curvature $l_{\Lambda_{E W}} \sim \frac{1}{4} \mathrm{~cm}$. So, $E_{\Lambda_{E W}} \sim 10^{-4} \mathrm{eV}$. For high energy experiments of order $20 \mathrm{TeV}$, one finds $E_{\Lambda_{T e V}} \sim 1 \mathrm{eV}$. Finally, for energies of order $1000 \mathrm{TeV}$, the value of $E_{\Lambda} \sim 2500 \mathrm{eV}$. For particles of small mass such as neutrinos, these deviations from the standard rest energy will lead to significant changes in kinematics at very high energies. It may be possible to test this proposal in not too distant a future.

Acknowledgments: This work was supported, in part by the Department of Energy under the contract number DOE-FGO2-84ER40153. I would like to thank Philip Argyres for reading the manuscript and making valuable suggestions for improvement. I am also indebted to Professor Arno Bohm for discussion and correspondence concerning his work.

\section{References}


[1] S. Weinberg, Rev. Mod. Phys. 61 (1989) 1.

[2] S. Carroll, astro-ph/0004075.

[3] Earlier versions of this work were reported at CPT01, International Conference on Tests of CPT and Lorentz Symmetry, August 2001, Bloomington, IN, and at XXXth Coral Gables Conference, December 2001, Fort Lauderdale, FL.

[4] F. Gürsey, in Group Theoretical Concepts and Methods in Elementary Particle Physics, ed. F. Gürsey, Gordon and Breach, 1962; F. Gürsey, in Relativity, Groups, and Topology, Les Houches Summer School 1963, Gordon and Breach, 1963; F. Gürsey and T.D. Lee, Pro. Natl. Acad. Sci. USA, 49 (1963) 179.

[5] C. Fronsdal, Rev. Mod. Phys. 37 (1965); Phys. Rev. D10 (1974) 589; Phys. Rev. D12 (1975) 3819; C. Fronsdal and R.B. Haugen, Phys. Rev. D12 (1975) 3810.

[6] N.T. Evans, Jour. Math. Phys. 8 (1967) 170

[7] L.H. Thomas, Ann. Math 42 (1941) 113; T.D. Newton, Ann. Math. 51 (1950) 730; J. Dixmier, Bull. Soc. Math. France 89 (1961) 9.

[8] A. Bohm, in Studies in Mathematical Physics, ed. A.O. Barut, Reidel Publ. Boston, 1973.

[9] E. A. Tagirov, Ann. Phys., 76 (1973) 561.

[10] S.J. Avis, C.J. Isham, D. Storey, Phys. Rev. D 18 (1978) 3565.

[11] E. Mottola, Phys. Rev. D 31 (1985) 754.

[12] B. Allen, Phys. Rev. D 32 (1985) 3136.

[13] For overviews of various theoretical ideas, see, for example, CPT and Lorentz Symmetry, ed. V.A. Kostelecky, World Scientific, Singapore, 1999.

[14] S. Fernando and F. Mansouri, Int. Jour. Mod. Phys. A 14 (1999) 505; [hepth/9804]. 